\title{
The Rabi model gives off a flavor of spontaneous SUSY breaking
}

\author{
Masao Hirokawa
}

Received: 4 November 2014 / Accepted: 21 April 2015 / Published online: 8 May 2015

(C) Chapman University 2015

\begin{abstract}
In this paper, we handle a two-level atom and a one-mode light primarily in SUSY state, and point out that an interaction between them brings them on a path to spontaneous SUSY breaking. That is, we prove that the Rabi model describing a qubit coupled with a one-mode light in a cavity takes the $N=2$ SUSY system to the spontaneous SUSY breaking as its coupling strength $\mathrm{g}$ grows larger from the case $\mathrm{g}=0$ to the case $\mathrm{g} \approx \infty$. We show that the spontaneous SUSY breaking is caused by the spin-chirality of the qubit, while the Rabi model recovers the chiral symmetry in the process of the growth of the coupling strength.
\end{abstract}

Keywords Spontaneous SUSY breaking · Rabi model

\section{Introduction}

Supersymmetric quantum mechanics (SUSYQM) was initiated by Witten [1,2], and has been developed (see Refs. [3,4] and the related papers in their references). In Refs. [5-10], several ground state structures and the spontaneous supersymmetry (SUSY) breaking were investigated. We are interested in a ground state structure in SUSYQM from another point of view than their preceding studies. We will handle the Rabi model that has the interaction between a two-level atom and a one-mode light in a cavity. The Rabi model has been well studied in quantum optics, and its some inherent properties have been beginning to be experimentally observed in cavity quantum electrodynamics (QED) and circuit QED [11-20].

The interaction between an atom and light in nature follows QED. It is governed by the fine-structure constant $\alpha \approx 0.00729735$, belonging to the region over which the perturbation theory is valid. On the other hand, cavity QED handles stronger interaction than the standard QED does [11,12]. Such a strong interaction is experimentally prepared with the coupling of a two-level atom and a one-mode light (i.e., single-mode laser) in a mirror cavity (i.e., a mirror resonator). Several solid-state analogs of the strong coupling had been foreseen in superconducting systems [13-15]. In short, we respectively replace the atom, the light, and the mirror resonator in cavity QED by an

The author acknowledges the support from JSPS, Grant-in-Aid for Scientific Research (B) 26310210 and Grant-in-Aid for Scientific Research (C) 26400117.

M. Hirokawa $(\varangle)$

Laboratory of Mathematics, Institute of Engineering, Hiroshima University, Hiroshima 739-8527, Japan

e-mail: hirokawa@ amath.hiroshima-u.ac.jp 
Fig. 1 Energy levels of $H_{\text {Rabi }}$ for $\omega:=\omega_{\mathrm{a}}=\omega_{\mathrm{c}}$. Each sort of lines indicates the $n$th level of the energy for $n=0,1,2, \ldots$ from the bottom

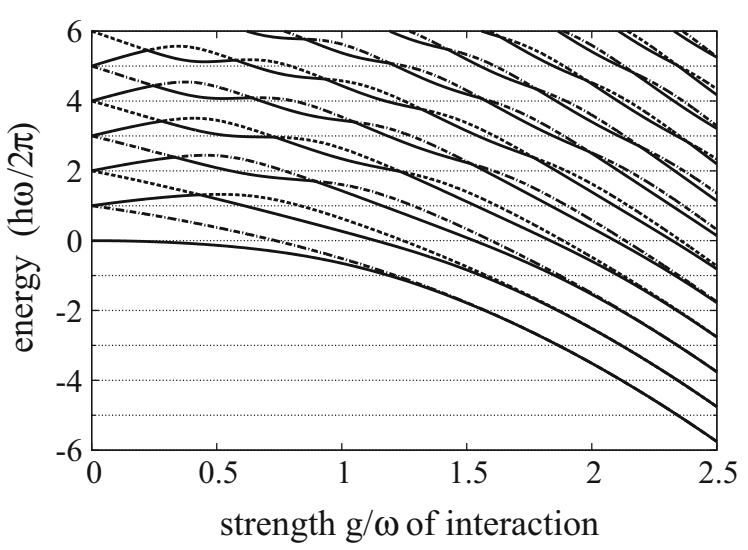

artificial atom, a microwave, and a microwave resonator on a superconducting circuit. The artificial atom consists of a superconducting circuit based on some Josephson junctions then. This replaced cavity QED is circuit QED, which has been experimentally demonstrated [16-18]. It is remarkable that circuit QED has been capable of intensifying the coupling strength further than cavity QED has $[19,20]$.

In this paper, we take an interest in a role of the Rabi model's interaction between the qubit of two-level atom and the one-mode light in the cavity from the point of the view of a SUSYQM aspect. We will show that its interaction brings the two-level atom and the one-mode light, which are primarily in SUSY state, on a path to spontaneous SUSY breaking. We will also show that this spontaneous SUSY breaking is caused by the spin-chirality between the two levels of the atom, while the Rabi model recovers the chiral symmetry as the coupling strength grows larger and larger.

\section{Rabi model}

We denote the annihilation (resp. creation) operator for the one-mode photon by $a$ (resp. $a^{\dagger}$ ). We use the standard notation for the Pauli matrices as $\sigma_{x} \equiv\left(\begin{array}{cc}0 & 1 \\ 1 & 0\end{array}\right), \sigma_{y} \equiv\left(\begin{array}{cc}0 & -i \\ i & 0\end{array}\right)$, and $\sigma_{z} \equiv\left(\begin{array}{cc}1 & 0 \\ 0 & -1\end{array}\right)$. We define spin states $|\uparrow\rangle$ and $|\downarrow\rangle$ by $|\uparrow\rangle:=\left(\begin{array}{l}1 \\ 0\end{array}\right)$ and $|\downarrow\rangle:=\left(\begin{array}{l}0 \\ 1\end{array}\right)$. We denote by $\mathcal{F}$ the Fock space of the one-mode photon, and by $|n\rangle$ the Fock state with the photon number $n=0,1,2, \ldots$ So, in particular, $|0\rangle$ denotes the Fock vacuum. Every quantum state that we will use in this paper is represented as $|n, \sharp\rangle:=|n\rangle|\sharp\rangle$ for $n=0,1,2, \ldots$ and $\sharp=\uparrow, \downarrow$. Here, we omitted the tensor-notation $\otimes$ from the expression $|n\rangle \otimes|\sharp\rangle$. We will use this omitted notation throughout this paper. Also, we denote by $|\psi, \sharp\rangle$ the state $\psi \otimes|\sharp\rangle$ for the state $\psi$ in the Fock space $\mathcal{F}$. Let us give the subspace $\mathcal{H}_{\text {even }}$ (resp. $\mathcal{H}_{\text {odd }}$ ) as the set of all of the states $|\psi, \uparrow\rangle$ (resp. $|\psi, \downarrow\rangle$ ), where the state $\psi$ runs over the whole Fock space. Then, the state space $\mathcal{H}:=\mathbb{C}^{2} \otimes \mathcal{F}$ is obviously decomposed as the direct sum of $\mathcal{H}_{\text {even }}$ and $\mathcal{H}_{\text {odd }}: \mathcal{H}=\mathcal{H}_{\text {even }} \oplus \mathcal{H}_{\text {odd }}$.

The free Hamiltonian $H_{0}$ of the Rabi model is given by

$H_{0}:=\frac{\hbar \omega_{\mathrm{a}}}{2} \sigma_{z}+\hbar \omega_{\mathrm{c}}\left(a^{\dagger} a+\frac{1}{2}\right)$.

The constants $\omega_{\mathrm{a}}$ and $\omega_{\mathrm{c}}$ are, respectively, the atom transition frequency and the cavity resonance frequency. Then, the Rabi Hamiltonian $H_{\text {Rabi }}$ is given by

$H_{\text {Rabi }}:=H_{0}+\hbar \mathrm{g}\left(a+a^{\dagger}\right) \sigma_{x}$,

where the parameter $\mathrm{g} \geq 0$ stands for the atom-photon coupling constant that represents the coupling strength. The solvability of the Rabi Hamiltonian has been argued by Braak [21], using Bargmann's representation [22]. Here, we give a numerical computation of the energies of the Rabi model in the case $\omega:=\omega_{\mathrm{a}}=\omega_{\mathrm{c}}$ in Fig. 1 .

Figure 1 attracts our particular attention to the two special cases, $g=0$ and $g \approx \infty$, in the light of SUSYQM. In the case $\mathrm{g}=0$, the ground state is unique, but all the excited states are twofold degenerate. All the eigenenergies 
line up at an equal interval $\hbar \omega$. Meanwhile, in case $g \approx \infty$, Fig. 1 makes us expect that all the states are almost twofold degenerate and all the eigenenergies are aligned at an almost equal interval $\hbar \omega$ as they diverge to negative infinity making the common shape of the energy curve, $-c_{0} \mathrm{~g}^{2}$ with a constant $c_{0}$. The energy with this shape must be used for the energy renormalization. We investigate these physical situations in detail from the point of the view of SUSYQM in this paper. So, for the condition, $\omega:=\omega_{\mathrm{a}}=\omega_{\mathrm{c}}$ we denote the free Hamiltonian $H_{0}$ by $H_{\mathrm{SS}}$ :

$H_{\mathrm{SS}}:=\frac{\hbar \omega}{2} \sigma_{z}+\hbar \omega\left(a^{\dagger} a+\frac{1}{2}\right)$.

We note that it is easy to tune the two frequencies, $\omega_{\mathrm{a}}$ and $\omega_{\mathrm{c}}$, in circuit QED so that they are equal.

\section{$3 \operatorname{SUSYQM}(\mathrm{g}=\mathbf{0})$}

First, when there is no interaction between the (artificial) atom and the light (i.e., g = 0), the Rabi Hamiltonian becomes the free Hamiltonian, $H_{\mathrm{Rabi}}=H_{\mathrm{SS}}$. So, it is the most popular Hamiltonian in SUSYQM:

$H_{\mathrm{SS}}=(1 / 2 m)\left(p^{2}+W^{2}+\hbar \frac{\mathrm{d} W}{\mathrm{~d} x} \sigma_{z}\right)$

with the correspondence,

$\left\{\begin{array}{l}a=\sqrt{m \omega / 2 \hbar} x+i p / \sqrt{2 m \hbar \omega}, \\ a^{\dagger}=\sqrt{m \omega / 2 \hbar} x-i p / \sqrt{2 m \hbar \omega},\end{array}\right.$

for the position operator $x$ and the momentum operator $p$, where the superpotential $W$ is given by $W(x)=m \omega x$. Namely, the system has $N=2$ SUSY. More precisely, the supercharges $Q_{1}$ and $Q_{2}$ defined by

$Q_{1}=(1 / 2 \sqrt{m})\left(W \sigma_{x}-p \sigma_{y}\right)$

and

$Q_{2}=(1 / 2 \sqrt{m})\left(W \sigma_{y}+p \sigma_{x}\right)$

make the relations,

$\left\{\begin{array}{l}\left\{Q_{k}, Q_{\ell}\right\}=\delta_{k \ell} H_{\text {Rabi }}, \\ {\left[Q_{k}, H_{\text {Rabi }}\right]=0,} \\ \left\{Q_{k}, N_{\mathrm{F}}\right\}=0,\end{array}\right.$

for $k, \ell=1,2$. The grading operator $N_{\mathrm{F}}=\sigma_{z}$ satisfying the conditions, $N_{\mathrm{F}} \psi_{\text {even }}=\psi_{\text {even }}$ for any state $\psi_{\text {even }} \in$ $\mathcal{H}_{\text {even }}$, and $N_{\mathrm{F}} \psi_{\text {odd }}=-\psi_{\text {odd }}$ for any state $\psi_{\text {odd }} \in \mathcal{H}_{\text {odd }}$. Here, the symbol $\delta_{k \ell}$ is the Kronecker delta. Then, the system has no SUSY breaking. That is, the supersymmetric (SUSY) ground state is $|0, \downarrow\rangle$ and therefore the ground state energy is equal to zero in this case. In addition, for $\mathrm{g}=0$, the ground state of the Rabi Hamiltonian is unique, but all its excited states are twofold degenerate. We can grasp these facts using the SUSY-generating charges $Q^{+}$ and $Q^{-}$defined by

$Q^{+}:=\sqrt{\hbar \omega} a \sigma_{+}$

and

$Q^{-}:=\sqrt{\hbar \omega} a^{\dagger} \sigma_{-}$,

where $\sigma_{-}$and $\sigma_{+}$are the spin annihilation and creation operators given by $\sigma_{ \pm}:=\left(\sigma_{x} \pm i \sigma_{y}\right) / 2$. The two degenerate excited states are interchanged with each other by the SUSY-generating charges $Q^{+}$and $Q^{-}$then: 


$$
\begin{aligned}
Q^{-}|n, \downarrow\rangle & =Q^{+}|n, \uparrow\rangle=0, \\
|n, \uparrow\rangle & =\frac{1}{\sqrt{\hbar \omega(n+1)}} Q^{+}|n+1, \downarrow\rangle, \\
|n+1, \downarrow\rangle & =\frac{1}{\sqrt{\hbar \omega(n+1)}} Q^{-}|n, \uparrow\rangle,
\end{aligned}
$$

for $n=0,1,2, \ldots$ As is well known, of course, we have the relations,

$$
\left\{\begin{array}{l}
\left\{Q^{ \pm}, Q^{ \pm}\right\}=0, \\
H_{\text {Rabi }}=\left\{Q^{+}, Q^{-}\right\} .
\end{array}\right.
$$

These relations that the SUSY-generating charges have explain the alignment of the eigenenergies of the Rabi model as the coupling constant is equal to zero.

\section{Spontaneous SUSY breaking $(\mathrm{g} \approx \infty)$}

In this section, let us take the coupling strength g large enough. We expect that the photon part energy,

$$
H_{\text {asym }}:=\hbar \omega\left(a^{\dagger} a+\frac{1}{2}\right)+\hbar \mathrm{g}\left(a+a^{\dagger}\right) \sigma_{x},
$$

is asymptotically much more dominant than the two-level atom energy $\hbar \omega \sigma_{z} / 2$. Namely, the atom energy $\hbar \omega \sigma_{z} / 2$ works as a very small perturbation for the photon part energy $H_{\text {asym }}$ around the coupling strength $\mathrm{g} \approx \infty$. We will show this in an exact way now. We define a unitary operator $U_{\mathrm{g}}$ by $U_{\mathrm{g}}:=\frac{1}{\sqrt{2}}\left(\begin{array}{cc}V_{-} & -V_{+} \\ V_{-} & V_{+}\end{array}\right)$with the unitary operator $V_{ \pm}:=e^{ \pm \mathrm{g}\left(a^{\dagger}-a\right) / \omega}$. Recall the well-known Bogoliubov transformation:

$V_{ \pm}\left\{\hbar \omega\left(a^{\dagger} a+\frac{1}{2}\right) \pm \hbar \mathrm{g}\left(a+a^{\dagger}\right)\right\} V_{\mp}=\hbar \omega\left(a^{\dagger} a+\frac{1}{2}\right)-\hbar \frac{\mathrm{g}^{2}}{\omega}$.

We then reach the unitary transformation,

$$
U_{\mathrm{g}}^{*} H_{\mathrm{Rabi}} U_{\mathrm{g}}=U_{\mathrm{g}}^{*} H_{\mathrm{asym}} U_{\mathrm{g}}-\frac{\hbar \omega}{2} \widetilde{V}_{\mathrm{g}}=\widetilde{H}_{0}-\hbar \frac{\mathrm{g}^{2}}{\omega}-\frac{\hbar \omega}{2} \widetilde{V}_{\mathrm{g}}
$$

with the free Hamiltonian

$$
\widetilde{H}_{0}=\left(\begin{array}{cc}
\hbar \omega\left(a^{\dagger} a+\frac{1}{2}\right) & 0 \\
0 & \hbar \omega\left(a^{\dagger} a+\frac{1}{2}\right)
\end{array}\right)
$$

and the unitary, self-adjoint interaction

$$
\widetilde{V}_{\mathrm{g}}=\left(\begin{array}{cc}
0 & e^{2 \mathrm{~g}\left(a^{\dagger}-a\right) / \omega} \\
e^{-2 \mathrm{~g}\left(a^{\dagger}-a\right) / \omega} & 0
\end{array}\right) .
$$

For arbitrary wave functions $\psi=\left(\begin{array}{l}\psi_{1} \\ \psi_{2}\end{array}\right)$ and $\phi=\left(\begin{array}{l}\phi_{1} \\ \phi_{2}\end{array}\right)$ in the state space $\mathcal{H}$, we set their unitary transformations as $\widetilde{\psi}_{j}:=e^{i \pi a^{\dagger} a / 2} \psi_{j}$ and $\widetilde{\phi}_{j}:=e^{i \pi a^{\dagger} a / 2} \phi_{j}$. Using the equations,

$\left\{\begin{array}{l}e^{i \pi a^{\dagger} a / 2} a e^{-i \pi a^{\dagger} a / 2}=-i a, \\ e^{i \pi a^{\dagger} a / 2} a^{\dagger} e^{-i \pi a^{\dagger} a / 2}=i a^{\dagger},\end{array}\right.$ 
we have

$$
\begin{aligned}
\left\langle\psi\left|\widetilde{V}_{\mathrm{g}}\right| \phi\right\rangle & =\left\langle\psi_{1} \mid e^{2 \mathrm{~g}\left(a^{\dagger}-a\right) / \omega} \phi_{2}\right\rangle+\left\langle\psi_{2} \mid e^{-2 \mathrm{~g}\left(a^{\dagger}-a\right) / \omega} \phi_{1}\right\rangle \\
& =\left\langle\widetilde{\psi}_{1} \mid e^{i(2 \mathrm{~g} / \omega)\left(a^{\dagger}+a\right)} \widetilde{\phi}_{2}\right\rangle+\left\langle\widetilde{\psi}_{2} \mid e^{-i(2 \mathrm{~g} / \omega)\left(a^{\dagger}+a\right)} \widetilde{\phi}_{1}\right\rangle .
\end{aligned}
$$

Since we have $a^{\dagger}+a=\sqrt{2 m \omega / \hbar} x$, we obtain the representation:

$\left\langle\psi\left|\widetilde{V}_{\mathrm{g}}\right| \phi\right\rangle=\int d x \widetilde{\psi}_{1}^{*}(x) \widetilde{\phi}_{2}(x) e^{i(2 \mathrm{~g} \sqrt{2 m / \hbar \omega}) x}+\int d x \widetilde{\psi}_{2}^{*}(x) \widetilde{\phi}_{1}(x) e^{-i(2 \mathrm{~g} \sqrt{2 m / \hbar \omega}) x}$.

The Riemann-Lebesgue's theorem, described in Theorem IX.7 of [23] for instance, tells us that the term $\left\langle\psi\left|\widetilde{V}_{\mathrm{g}}\right| \phi\right\rangle$ vanishes as $\lim _{\mathrm{g} \rightarrow \infty}\left\langle\psi\left|\widetilde{V}_{\mathrm{g}}\right| \phi\right\rangle=0$. More precisely, we can explain this decay in the following: We set the parameter $2 \mathrm{~g} \sqrt{2 m / \hbar \omega}$ as $G$, i.e., $G:=2 \mathrm{~g} \sqrt{2 m / \hbar \omega}$. We denote the Fourier transformation of the multiplied function $\widetilde{\psi}_{j}^{*}(x) \widetilde{\phi}_{k}(x)$ by $f(\xi)$, i.e., $f(\xi):=\int \mathrm{d} x \widetilde{\psi}_{j}^{*}(x) \widetilde{\phi}_{k}(x) e^{-i \xi x}$. Since we have the equation $e^{-i \xi x}=-e^{-i \xi(x-\pi / \xi)}$, we can rewrite the function $f(\xi)$ as

$f(\xi):=-\int \mathrm{d} x \widetilde{\psi}_{j}^{*}(x+\pi / \xi) \widetilde{\phi}_{k}(x+\pi / \xi) e^{-i \xi x}$,

where we used the integration by substitution, $x-\pi / \xi \rightarrow x$. Thus, we obtain the equation,

$f(G)=\frac{1}{2} \int \mathrm{d} x\left\{\widetilde{\psi}_{j}^{*}(x) \widetilde{\phi}_{k}(x)-\widetilde{\psi}_{j}^{*}(x+\pi / G) \widetilde{\phi}_{k}(x+\pi / G)\right\} e^{-i G x}$,

which implies the inequality,

$|f(G)| \leq \frac{1}{2} \int \mathrm{d} x\left|\widetilde{\psi}_{j}^{*}(x) \widetilde{\phi}_{k}(x)-\widetilde{\psi}_{j}^{*}(x+\pi / G) \widetilde{\phi}_{k}(x+\pi / G)\right|$.

Since the multiplied function $\widetilde{\psi}_{j}^{*}(y) \widetilde{\phi}_{k}(y)$ is integrable, the Lebesgue's dominated convergence theorem, together with the approximation argument for the function $\widetilde{\psi}_{j}^{*}(y) \widetilde{\phi}_{k}(y)$ with a proper continuous function, says that the function $|f(G)|$ decays as the parameter $G$ tends to the infinity.

This decay supplies us with the convergence of the operators $U_{\mathrm{g}}^{*}\left(H_{\mathrm{Rabi}}+\hbar \mathrm{g}^{2} / \omega\right) U_{\mathrm{g}}$ in the following weak sense:

$\lim _{\mathrm{g} \rightarrow \infty}\left\langle\psi\left|U_{\mathrm{g}}^{*}\left(H_{\text {Rabi }}+\hbar \mathrm{g}^{2} / \omega\right) U_{\mathrm{g}}\right| \phi\right\rangle=\left\langle\psi\left|U_{\mathrm{g}}^{*}\left(H_{\mathrm{asym}}+\hbar \mathrm{g}^{2} / \omega\right) U_{\mathrm{g}}\right| \phi\right\rangle=\left\langle\psi\left|\widetilde{H}_{0}\right| \phi\right\rangle$

for arbitrary wave functions $\psi$ and $\phi$ in the domain of the operator $\widetilde{H}_{0}$. We point out that the vector $U_{\mathrm{g}}^{*}\left(H_{\mathrm{Rabi}}+\right.$ $\left.\hbar \mathrm{g}^{2} / \omega\right) U_{\mathrm{g}}|\phi\rangle$ never converges to the vector $\widetilde{H}_{0}|\phi\rangle$ in the sense of the norm convergence due to the equation, $\left\langle\psi\left|\widetilde{V}_{\mathrm{g}}^{*} \widetilde{V}_{\mathrm{g}}\right| \psi\right\rangle=\langle\psi \mid \psi\rangle$, where the norm is induced by the inner product of the Hilbert space $\mathcal{H}$.

From now on, we will prove that the weak convergence (5) induces spontaneous SUSY breaking, which makes a correspondence between eigenstates $\varphi^{\text {Rabi }}$ of the Rabi Hamiltonian $H_{\text {Rabi }}$ and eigenstates $\varphi_{n}$ of the asymptotic Hamiltonian $H_{\text {asym }}$ in the following:

$\varphi^{\text {Rabi }} \approx \varphi_{n}, \quad \mathrm{~g} \gg 1$,

where $n$ is the non-negative integer satisfying the condition for the eigenenergy $E^{\text {Rabi }}$ of the eigenstate $\varphi^{\text {Rabi }}$ :

$E^{\mathrm{Rabi}} \approx \hbar \omega\left(n+\frac{1}{2}\right)-\hbar \frac{\mathrm{g}^{2}}{\omega}, \quad \mathrm{g} \gg 1$.

Actually, we can chose the eigenstate $\varphi_{n}$ as either of one of eigenvectors $U_{\mathrm{g}}|n, \uparrow\rangle$ and $U_{\mathrm{g}}|n, \downarrow\rangle$. Equations (6) and (7) show asymptotically twofold degenerate energy levels as in Fig. 1. 
We here recall that the Rabi Hamiltonian has the following parity symmetry: $\left[H_{\mathrm{Rabi}}, \Pi\right]=0$ for the parity operator $\Pi:=\sigma_{z}(-1)^{a^{\dagger} a}$. So, adopting the representations $b:=\sigma_{x} a$ and $b^{\dagger}:=\sigma_{x} a^{\dagger}$ satisfying the canonical commutation relation, $\left[b, b^{\dagger}\right]=1$, the Rabi Hamiltonian has the expression $H_{\text {Rabi }}=H_{\text {asym }}+\hbar \omega \sigma_{z} / 2$ with

$H_{\text {asym }}=\hbar \omega\left(b^{\dagger} b+\frac{1}{2}\right)+\hbar \mathrm{g}\left(b^{\dagger}+b\right)$

and $\frac{\hbar \omega}{2} \sigma_{z}=\frac{\hbar \omega}{2}(-1)^{b^{\dagger} b} \Pi \longrightarrow 0$ as $\mathrm{g} \rightarrow \infty$ in the weak sense by Eq. (5). Equation (8) tells us what is the energy that we have to use for renormalization. Thus, based on this expression, we define the asymptotically renormalized (AR) Rabi Hamiltonian $H_{\mathrm{Rabi}}^{\mathrm{AR}}$ by

$H_{\mathrm{Rabi}}^{\mathrm{AR}}:=H_{\mathrm{asym}}+\hbar \frac{\mathrm{g}^{2}}{\omega}$.

Then, the weak convergence Eq. (5) tells us that the energy-renormalized Rabi Hamiltonian $H_{\text {Rabi }}+\hbar \frac{\mathrm{g}^{2}}{\omega}$ converges to the AR Rabi Hamiltonian $H_{\mathrm{Rabi}}^{\mathrm{AR}}$ as $\mathrm{g} \rightarrow \infty$ in the weak sense:

$H_{\mathrm{Rabi}}+\hbar \frac{\mathrm{g}^{2}}{\omega} \underset{\mathrm{g} \rightarrow \infty}{\mathrm{w}} H_{\mathrm{Rabi}}^{\mathrm{AR}}$.

In mathematical terms, Eq. (5) implies that the energy-renormalized Rabi Hamiltonian $H_{\text {Rabi }}+\hbar \mathrm{g}^{2} / \omega$ converges to the AR Rabi Hamiltonian $H_{\mathrm{Rabi}}^{\mathrm{AR}}$ in the strong resolvent sense:

$H_{\text {Rabi }}+\hbar \frac{\mathrm{g}^{2}}{\omega} \underset{\mathrm{g} \rightarrow \infty}{\stackrel{\text { s.r.s. }}{\longrightarrow}} H_{\mathrm{Rabi}}^{\mathrm{AR}}$.

For the definition of the convergence of operators in strong resolvent sense, see Definition on p. 284 of Ref. [24]. We here prove Eq. (10) briefly. We denote the operators $H_{\mathrm{Rabi}}+\hbar \frac{\mathrm{g}^{2}}{\omega}$ and $H_{\mathrm{Rabi}}^{\mathrm{AR}}$ by $A_{\mathrm{g}}$ and $A$, respectively. Using the second resolvent equation, we have

$\left(i+A_{\mathrm{g}}\right)^{-1}-(i+A)^{-1}=\left(i+A_{\mathrm{g}}\right)^{-1}\left(A-A_{\mathrm{g}}\right)(i+A)^{-1}$.

Thus, the above equation leads to the following equation:

$\left(i+A_{\mathrm{g}}\right)^{-1}-(i+A)^{-1}=\left(i+A_{\mathrm{g}}\right)^{-1}(i+A)(i+A)^{-1}\left(A-A_{\mathrm{g}}\right)(i+A)^{-1}$.

In addition, by Eqs. (4) and (11), we have

$$
\begin{aligned}
\left(i+A_{\mathrm{g}}\right)^{-1}(i+A) & =\left\{\left(i+A_{\mathrm{g}}\right)^{-1}\left(A-A_{\mathrm{g}}\right)(i+A)^{-1}+(i+A)^{-1}\right\}(i+A) \\
& =\left(i+A_{\mathrm{g}}\right)^{-1}\left(A-A_{\mathrm{g}}\right)+1 \\
& =\frac{\hbar \omega}{2}\left(i+A_{\mathrm{g}}\right)^{-1} U_{\mathrm{g}} \widetilde{V}_{\mathrm{g}} U_{\mathrm{g}}^{*}+1 .
\end{aligned}
$$

Thus, for the operator norm \|\|$_{\text {op }}$, we obtain

$$
\left\|\left(i+A_{\mathrm{g}}\right)^{-1}(i+A)\right\|_{\mathrm{op}} \leq \frac{\hbar \omega}{2}\left\|\left(i+A_{\mathrm{g}}\right)^{-1}\right\|_{\mathrm{op}}\left\|U_{\mathrm{g}} \widetilde{V}_{\mathrm{g}} U_{\mathrm{g}}^{*}\right\|_{\mathrm{op}}+1 \leq \frac{\hbar \omega}{2}+1 .
$$

Here, for the definition of the operator norm, see Example 4 in §III of Ref. [24]. We note that the operator $(i+A)^{-1}$ is compact (or completely continuous). For the exact definition of a compact operator, see Definition on p. 199 of Ref. [24]. The reason why our operator $(i+A)^{-1}$ becomes compact follows from the equivalence of (ii) and (v) of Theorem XIII.64 of Ref. [25]. We also note that Eq. (5) implies that the term $\left(A-A_{\mathrm{g}}\right)(i+A)^{-1} \varphi$ weakly converges to 0 for every state $\varphi$, i.e.,

$\lim _{\mathrm{g} \rightarrow \infty}\left\langle\psi \mid\left(A-A_{\mathrm{g}}\right)(i+A)^{-1} \varphi\right\rangle=0$ 
for all states $\varphi, \psi$ in the state space $\mathbb{C}^{2} \otimes \mathcal{F}$. Thus, Theorem VI.11 of Ref. [24] tells us the convergence,

$$
\lim _{\mathrm{g} \rightarrow \infty}\left\|(i+A)^{-1}\left(A-A_{\mathrm{g}}\right)(i+A)^{-1} \varphi\right\|_{\mathbb{C}^{2} \otimes \mathcal{F}}=0,
$$

where the norm $\|\cdot\|_{\mathbb{C}^{2} \otimes \mathcal{F}}$ is of the state space $\mathbb{C}^{2} \otimes \mathcal{F}$ given by $\|\cdot\|_{\mathbb{C}^{2} \otimes \mathcal{F}}=\langle\cdot \mid \cdot\rangle^{1 / 2}$. Combining Eqs. (12)-(14), we reach the strong resolvent convergence of operators $A_{\mathrm{g}}$ to the operator $A$ as $\mathrm{g} \rightarrow \infty$.

Therefore, Eqs. (6) and (7) can also be obtained by the method of spectral analysis in mathematics by applying Theorem VIII.24 of Ref. [24] to our case, but we prove them in the light of SUSYQM in this paper.

We define the system's supercharges $Q_{1}$ and $Q_{2}$ by

$Q_{1}:=U_{\mathrm{g}} \widetilde{Q}_{1} U_{\mathrm{g}}^{*}$

and

$Q_{2}:=U_{\mathrm{g}} \widetilde{Q}_{2} U_{\mathrm{g}}^{*}$,

where the operators $\widetilde{Q}_{1}$ and $\widetilde{Q}_{2}$ are given by

$$
\widetilde{Q}_{1}:=\sqrt{\frac{\hbar \omega}{2}} \sqrt{a^{\dagger} a+\frac{1}{2}} \sigma_{x}
$$

and

$$
\widetilde{Q}_{2}:=\sqrt{\frac{\hbar \omega}{2}}\left(-i \sqrt{a^{\dagger} a+\frac{1}{2}} \sigma_{+}+i \sqrt{a^{\dagger} a+\frac{1}{2}} \sigma_{-}\right) .
$$

Then, we have the relations,

$$
\left\{\begin{array}{l}
\left\{Q_{k}, Q_{\ell}\right\}=\delta_{k \ell} H_{\mathrm{Rabi}}^{\mathrm{AR}}, \\
{\left[Q_{k}, H_{\mathrm{Rabi}}^{\mathrm{AR}}\right]=0,} \\
\left\{Q_{k}, N_{\mathrm{F}}\right\}=0,
\end{array}\right.
$$

for $k, \ell=1,2$. The grading operator $N_{\mathrm{F}}=U_{\mathrm{g}} \sigma_{z} U_{\mathrm{g}}^{*}=\sigma_{x}$, which satisfies the conditions, $N_{\mathrm{F}} \psi_{\text {even }}=\psi_{\text {even }}$ for any state $\psi_{\text {even }} \in U_{\mathrm{g}} \mathcal{H}_{\text {even }}$, and $N_{\mathrm{F}} \psi_{\text {odd }}=-\psi_{\text {odd }}$ for any state $\psi_{\text {odd }} \in U_{\mathrm{g}} \mathcal{H}_{\text {odd }}$. We note the equation concerning the whole state space, $\mathcal{H}=U_{\mathrm{g}} \mathcal{H}=\left(U_{\mathrm{g}} \mathcal{H}_{\text {even }}\right) \oplus\left(U_{\mathrm{g}} \mathcal{H}_{\text {odd }}\right)$. We have the SUSY-generating supercharges $Q^{-}$and $Q^{+}$as

$Q^{-}:=U_{\mathrm{g}} \widetilde{Q}^{-} U_{\mathrm{g}}^{*}$

and

$$
Q^{+}:=U_{\mathrm{g}} \widetilde{Q}^{+} U_{\mathrm{g}}^{*}
$$

where the operators $\widetilde{Q}^{-}$and $\widetilde{Q}^{+}$are given by

$$
\widetilde{Q}^{-}=\sqrt{\hbar \omega\left(a^{\dagger} a+\frac{1}{2}\right)} \sigma_{+}
$$

and

$$
\widetilde{Q}^{+}=\sqrt{\hbar \omega\left(a^{\dagger} a+\frac{1}{2}\right)} \sigma_{-} .
$$


The SUSY-generating supercharges $Q^{-}$and $Q^{+}$satisfy the relations,

$$
\left\{\begin{array}{l}
\left\{Q^{ \pm}, Q^{ \pm}\right\}=0, \\
H_{\mathrm{Rabi}}^{\mathrm{AR}}=\left\{Q^{+}, Q^{-}\right\} .
\end{array}\right.
$$

Then, the following relations explain the alignment of the eigenenergies of the Rabi model in the case the coupling constant is infinitely large:

$$
\begin{aligned}
Q^{-} U_{\mathrm{g}}|n, \uparrow\rangle & =Q^{+} U_{\mathrm{g}}|n, \downarrow\rangle=0, \\
U_{\mathrm{g}}|n, \downarrow\rangle & =\frac{1}{\sqrt{\hbar \omega(n+1 / 2)}} Q^{+} U_{\mathrm{g}}|n, \uparrow\rangle, \\
U_{\mathrm{g}}|n, \uparrow\rangle & =\frac{1}{\sqrt{\hbar \omega(n+1 / 2)}} Q^{-} U_{\mathrm{g}}|n, \downarrow\rangle,
\end{aligned}
$$

for $n=0,1,2, \ldots$ We note, in particular, that $Q^{+} U_{\mathrm{g}}|0, \uparrow\rangle=\sqrt{\hbar \omega / 2} U_{\mathrm{g}}|0, \downarrow\rangle \neq 0$ and $Q^{-} U_{\mathrm{g}}|0, \downarrow\rangle=$ $\sqrt{\hbar \omega / 2} U_{\mathrm{g}}|0, \uparrow\rangle \neq 0$, which shows spontaneous SUSY breaking as described below.

We here remember that the Pauli matrix $\sigma_{x}$ makes the spin-chiral transformation, $\sigma_{x}|\uparrow\rangle=|\downarrow\rangle$ and $\sigma_{x}|\downarrow\rangle=|\uparrow\rangle$. Using the equations, $\left[\sigma_{z}, \widetilde{H}_{0}\right]=0$ and $U_{\mathrm{g}} \sigma_{z} U_{\mathrm{g}}^{*}=\sigma_{x}$, we can show the chiral symmetry,

$$
\left[\sigma_{x}, H_{\mathrm{Rabi}}^{\mathrm{AR}}\right]=0 \text {, }
$$

though the Rabi Hamiltonian $H_{\text {Rabi }}$ does not have the chiral symmetry primarily:

$\left[\sigma_{x}, H_{\text {Rabi }}\right] \neq 0$.

Define the states $\psi_{+}$and $\psi_{-}$by $\psi_{ \pm}:=U_{\mathrm{g}}(|0, \uparrow\rangle \pm|0, \downarrow\rangle) / \sqrt{2}$. Then, the states $\psi_{+}$and $\psi_{-}$are the lowest-energy states of the AR Rabi Hamiltonian $H_{\mathrm{Rabi}}^{\mathrm{AR}}$. We then reach the fact that

$\sigma_{x} \psi_{+}=\psi_{-} \neq \psi_{+}$with $\left\langle\psi_{+} \mid \psi_{-}\right\rangle=0$.

Although the AR Rabi Hamiltonian $H_{\mathrm{Rabi}}^{\mathrm{AR}}$ has the spin-chiral symmetry (15), the lowest-energy state is not invariant under the spin-chirality as in the relation (17). This is exactly the spontaneous SUSY breaking that we are interested in. Thus, the system does not have the SUSY ground state, i.e., the lowest energy of the AR Rabi Hamiltonian is $\hbar \omega / 2$ and it is strictly positive.

\section{Conclusion and discussion}

From these arguments, we eventually realize that the growth of the coupling strength of the Rabi model plays a role of taking the $N=2$ SUSY to the spontaneous SUSY breaking, while the Rabi model gradually recovers the chiral symmetry as the coupling strength grows infinitely large. We have been interested in the process of the coupling strength's growth that breaks the SUSY. It is well known [26-28] that we can give a basis of a non-compact orthosymplectic superalgebra as

$$
\begin{aligned}
K_{+} & :=\frac{1}{2} a^{\dagger} a^{\dagger}, K_{-}:=\frac{1}{2} a a, \\
K_{0} & :=\frac{1}{4} a^{\dagger} a+\frac{1}{4}, B:=\frac{1}{4} \sigma_{z}, \\
W_{+}^{\mathrm{R}} & :=\frac{1}{\sqrt{2}} a^{\dagger} \sigma_{-}, W_{-}^{\mathrm{R}}:=\frac{1}{\sqrt{2}} a \sigma_{+}, \\
W_{+}^{\mathrm{CR}} & :=\frac{1}{\sqrt{2}} a^{\dagger} \sigma_{+}, W_{-}^{\mathrm{CR}}:=\frac{1}{\sqrt{2}} a \sigma_{-} .
\end{aligned}
$$


Then, our SUSY Hamiltonian $H_{\mathrm{SS}}$ is written as $H_{\mathrm{SS}}=2 \hbar \omega K_{0}+2 \hbar \omega B$, and the operators $W_{\mathrm{R}}$ and $W_{\mathrm{CR}}$, respectively, called the rotating term and the counter-rotating term in quantum optics, are given by

$W_{\mathrm{R}}:=\sqrt{2}\left(W_{+}^{\mathrm{R}}+W_{-}^{\mathrm{R}}\right)$,

$W_{\mathrm{CR}}:=\sqrt{2}\left(W_{+}^{\mathrm{CR}}+W_{-}^{\mathrm{CR}}\right)$.

They are, respectively, given by the spin-chiral transformation of each other: $W_{\mathrm{CR}}=\sigma_{x} W_{\mathrm{R}} \sigma_{x}$. While the rotating term $W_{\mathrm{R}}$ acts in the standard state space $\mathbb{C}^{2} \otimes \mathcal{F}$, the counter-rotating term $W_{\mathrm{CR}}$ becomes a rotating term acting in the chiral state space $\sigma_{x} \mathbb{C}^{2} \otimes \mathcal{F}$ which is, of course, mathematically equal to $\mathbb{C}^{2} \otimes \mathcal{F}$ itself. We can rewrite the Rabi Hamiltonian as

$H_{\mathrm{Rabi}}=H_{\mathrm{SS}}+\hbar \mathrm{g}\left(W_{\mathrm{R}}+\sigma_{x} W_{\mathrm{R}} \sigma_{x}\right)$.

The rotating term $W_{\mathrm{R}}$ and the chiral rotating term $\sigma_{x} W_{\mathrm{R}} \sigma_{x}$ are mathematically equitable in the interaction of the Rabi Hamiltonian. That is, both of them equally appear in the mathematical description of the Rabi model's interaction. But, actually, their individual roles depend on the coupling strength. According to Eqs. (9), (15), and (16), the growth of the coupling strength restores the chiral symmetry. Actually, some experimental results of cavity and circuit QEDs $[11,12,18]$, in the weak and strong coupling regimes the contribution by the chiral rotating term (i.e., the counter-rotating term) is so small that it can be negligible, and thus, the rotating wave approximation (RWA) works in spite of the loss of the original, mathematical equitableness. On the other hand, the effect of the chiral rotating term remarkably appears and plays an important role when the coupling strength plunges into a region beyond that strong coupling regime $[19,20]$. The region beyond the strong coupling regime is called the ultra-strong coupling regime in circuit QED [19,20,29-32]. Namely, the division between the regimes of strong and ultra-strong couplings is crucial to whether the chiral rotating term restores. The present technology of circuit QED has been beginning to show us the division, and their experimental results say that the mathematical equitableness is lost in the weak coupling regime, but the growth of the coupling strength tries to recover it in the ultra-strong coupling regime. We are conjecturing that the process to the spontaneous SUSY breaking recovers the mathematical equitableness concerning the spin-chirality in the Rabi model [33], and then, a chirality quantum phase transition [34] may be concerned in the process.

The conflict between the effects by the rotating term and the chiral rotating term reminds us of Hund's paradox on the chiral molecules [35-39], though the paradox is concerned with the states of molecule. There would be a possibility that circuit QED experimentally demonstrates a quantum simulation [40-42] for some problems on chirality such as explained in this paper.

\section{References}

1. Witten, E.: Dynamical breaking of supersymmetry. Nucl. Phys. B 188, 513 (1981)

2. Witten, E.: Constraints on supersymmetry breaking. Nucl. Phys. B 202, 253 (1982)

3. Gangopadhyaya, A., Mallow, J.V., Rasinariu, C.: Supersymmetric quantum mechanics. World Scientific, New Jersey (2011)

4. Schwabl, F.: Quantum mechanics. Springer, Berlin (2007)

5. Claudson, M., Halpern, M.: Supersymmetric ground state wave functions. Nucl. Phys. B 250, 689 (1985)

6. Jaffe, A., Lesniewski, A., Lewenstei, M.: Ground state structure in supersymmetric quantum mechanics. Ann. Phys. (NY) 178, 313 (1987)

7. Arai, A.: Supersymmetric embedding of a model of a quantum harmonic oscillator interacting with infinitely many bosons. J. Math. Phys. 30, 512 (1989)

8. Kuroki, T., Sugino, F.: Spontaneous supersymmetry breaking by large- $N$ matrices. Nucl. Phys. B 796, 471 (2008)

9. Kuroki, T., Sugino, F.: Spontaneous supersymmetry breaking in large- $N$ matrix models with slowly varying potential. Nucl. Phys. B 830, 434 (2010)

10. Kuroki, T., Sugino, F.: Spontaneous supersymmetry breaking in matrix models from the viewpoints of localization and Nicolai mapping. Nucl. Phys. B 844, 409 (2011)

11. Haroche, S., Raimond, J.M.: Exploring quantum. Atoms, cavities, and photons. Oxford University Press, Oxford (2008) 
12. Raimond, J.M., Brune, M., Harohe, S.: Manipulating quantum entanglement with atoms and photons in a cavity. Rev. Mod. Phys. 73, 565 (2001)

13. Marquardt, F., Bruder, C.: Superposition of two mesoscopically distinct quantum states: coupling a Cooper-pair box to a large superconducting island. Phys. Rev. B 63, 054514 (2001)

14. Makhilin, Yu., Schön, G., Shnirman, A.: Quantum-state engineering with Josephson-junction devices. Rev. Mod. Phys. 73, 357 (2001)

15. Blais, A., Huang, R.-S., Givrin, S., Schoelkopf, R.: Cavity quantum electrodynamics for superconducting electrical circuits: an architecture for quantum computation. Phys. Rev. A 69, 062320 (2004)

16. Chiorescu, I., Bertet, P., Semba, K., Nakamura, Y., Harmans, C.J.P.M., Mooij, J.E.: Coherent dynamics of a flux qubit coupled to a harmonic oscillator. Nature 431, 159 (2004)

17. Wallraff, A., Schuster, D.I., Blais, A., Fruzio, L., Huang, R.-S., Majer, J., Kuar, S., Girvin, S.M., Schoelkop, R.J.: Strong coupling of a single photon to a superconducting qubit using circuit quantum electrodynamics. Nature 431, 162 (2004)

18. Fink, J.M., Göppl, M., Baur, M., Bianchetti, R., Leek, P.J., Blais, A., Wallraff, A.: Climbing the Jaynes-Cummings ladder and observing its nonlinearity in a cavity QED system. Nature 454, 315 (2008)

19. Niemczyk, T., Deppe, F., Huebl, H., Menzel, E.P., Hocke, F., Schwarz, M.J., Garcia-Ripoll, J.J., Zueco, D., Hümmer, T., Solano, E., Marx, A., Gross, R.: Circuit quantum electrodynamics in the ultrastrong-coupling regime. Nat. Phys. 6, 772 (2010)

20. Forn-Díaz, P., Lisenfeld, J., Marcos, D., García-Ripoll, J.J., Solano, E., Harmans, C.J.P.M., Mooij, J.E.: Observation of the BlochSiegert shift in a qubit-oscillator system in the ultrastrong coupling regime. Phys. Rev. Lett. 105, 237001 (2010)

21. Braak, D.: Integrability of the Rabi model. Phys. Rev. Lett. 107, 100401 (2011)

22. Reik, H.G., Doucha, M.: Exact solution of the Rabi Hamiltonian by known functions? Phys. Rev. Lett. 57, 787 (1986)

23. Reed, M., Simon, B.: Methods of moderm mathematical physics II: fourier analysis, self-adjointness. Academic Press, New York (1975)

24. Reed, M., Simon, B.: Methods of moderm mathematical physics I: functional analysis. Academic Press, New York (1980)

25. Reed, M., Simon, B.: Methods of moderm mathematical physics IV: analysis of operators. Academic Press, New York (1978)

26. Balantekin, A.B.: Accidental degeneracies and supersymmetric quantum mechanics. Ann. Phys. (NY) 164, 277 (1985)

27. Schmitt, H.A., Mufti, A.: Two-level Jaynes-Cummings Hamiltonian as a supersymmetric quantum-mechanical system. Phys. Rev. D 43, 2743 (1991)

28. Kostelecky, V.A., Nieto, M.M., Truax, D.R.: Supersymmetry and the relationship between the Coulomb and oscillator problems in arbitrary dimensions. Phys. Rev. D 32, 2627 (1985)

29. Devoret, M., Girvin, S., Schoelkopf, R.: Circuit-QED: Howstrong can the coupling between a Josephson junction atom and a transmission line resonator be? Ann. Phys. (Leipzig) 16, 76 (2007)

30. Clarke, J., Wilhelm, F.K.: Superconducting quantum bits. Nature 453, 1031 (2008)

31. Günter, G., Anappara, A.A., Hees, J., Sell, A., Biasiol, G., Sorba, L., De Liberato, S., Ciuiti, C., Tredicucci, A., Leitenstorfer, A., Huber, R.: Sub-cycle switch-on of ultrastrong light-matter interaction. Nature 458, 178 (2009)

32. Casanova, J., Romera, G., Lizuain, I., García-Ripoll, J.J., Solano, E.: Deep strong coupling regime of the Jaynes-Cummings model. Phys. Rev. Lett. 105, 263603 (2010)

33. Hirokawa, M.: On the coupling-strength growth of the Rabi model in the light of SUSYQM. arXiv: 1101.1770v3

34. Bermudez, A., Martin-Delgado, M. A., Luis, A.: Chirality quantum phase transition in the Dirac oscillator. Phys. Rev. A 77, 063815 (2008)

35. Hund, F.: Zur Deutung der Molekelspektren. I. Z. Phys. 40, 742 (1927)

36. Hund, F.: Zur Deutung der Molekelspektren. II. Z. Phys. 42, 93 (1927)

37. Hund, F.: Zur Deutung der Molekelspektren. III. Z. Phys. 43, 805 (1927)

38. Wightman, A. S., Glance, N.: Superselection rules in molecules. Nucl. Phys. B (Proc. Suppl.) 6, 202 (1989)

39. Wightman, A.S.: Superselection rules; old and new. Nuovo Cimento B 110, 751 (1995)

40. Feynman, R.P.: Simulating physics with computers. Int. J. Theor. Phys. 21, 467 (1982)

41. Bermudez, A., Martin-Delgado, M. A., Solano, E.: Mesoscopic superposition states in relativistic Landau levels. Phys. Rev. Lett. 99, 123602 (2007)

42. Bermudez, A., Martin-Delgado, M. A., Solano, E.: Exact mapping of the $2+1$ Dirac oscillator onto the Jaynes-Cummings model: Ion-trap experimental proposal. Phys. Rev. A 76, 041801(R) (2007) 